\title{
Direct Conjugation of Semiconductor Nanocrystals to a Globular Protein to Study Protein-Folding Intermediates
}

\author{
Rupa Sarkar, ${ }^{\dagger}$ S. Shankara Narayanan, ${ }^{\dagger}$ Lars-Olof Pålsson, ${ }^{\dagger}$ Fernando Dias, ${ }^{\S}$ \\ Andy Monkman, $₫$ and Samir Kumar Pal*, ${ }^{\star}$ \\ Unit for Nano Science \& Technology, Department of Chemical, Biological \& Macromolecular Sciences, \\ S. N. Bose National Centre for Basic Sciences, Block JD, Sector III, Salt Lake, Kolkata 700 098, India, \\ and University of Durham, United Kingdom
}

Received: July 5, 2007; In Final Form: August 20, 2007

\begin{abstract}
In this Article, we study the development of semiconductor nanocrystals (quantum dots of average diameter less than $2 \mathrm{~nm}$ ) directly conjugated to a transporter protein human serum albumin (HSA) as fluorescent biological labels. Förster resonance energy transfer (FRET) from the amino acid tryptophan (Trp214) to quantum dot in HSA is monitored to follow the local and global changes in the protein structure during thermal unfolding and refolding processes. This study is likely to attract widespread attention as a powerful tool for the study of protein folding.
\end{abstract}

\section{Introduction}

In recent years, extensive experimental and theoretical efforts are underway to integrate nanotechnology with biology and medicine for development in molecular diagnostics, therapeutics, molecular biology, and bioengineering. ${ }^{1-3}$ The efficacy of nanoparticle for any application is strongly dependent upon their physiochemical characteristics and their interactions with surface modifiers. A variety of different surface passivating agents such as surfactants, amines, thiols, and phosphates have been used ${ }^{4}$ for the preparation of nanocrystals in the quantum size regime. However, selection of suitable protecting agents for biological applications demands dimensional stability, good solubility, environment friendly nature, better sensitivity, minimum invasiveness, good biocompatibility, and bioactive functionalities. ${ }^{5}$ Biosynthetic preparation technique involves incubation of biomolecules (protein, DNA) with nanoparticles. ${ }^{6,7}$ In a recent work, chemically modified protein has been used to coat the surface of water-soluble quantum dots (QD), to restore their fluorescence, and to provide functional groups for bioconjugation. ${ }^{8}$ Yet this technique has some disadvantages: (i) noncovalent labeling does not give any site-specific information of the biomolecules; (ii) the samples are generally stored at low temperature to impart stability, but possibility of aggregation increases at low temperature; and (iii) densely passivated surface does not allow any external species (e.g., drug molecule) to interact with the protected nanoparticle. An alternative nonconventional route involves direct conjugation of nanoparticle to biomolecules that provides an opportunity to study various structural and functional aspects of the biomolecule using nanoparticle as a probe/sensor. The covalent binding also eliminates the possibility of aggregation, the need of a linker molecule that densely passivates the nanoparticle surface and hinders the particle to use as a biosensor. A few reports ${ }^{9-11}$ are in the literature on the direct conjugation of nanoparticle to biomolecules. Recently, BSA-conjugated nanoparticle has been

\footnotetext{
* Corresponding author. Fax: 91332335 3477. E-mail: skpal@bose.res.in

†. N. Bose National Centre for Basic Sciences.

$\doteqdot$ Department of Chemistry, University of Durham.

$\S$ Department of Physics, University of Durham.
}

synthesized by expanding supercritical solution into a liquid solvent. ${ }^{9,10}$ This procedure requires high temperature and high pressure where the retention of structural integrity of the protein is not expected. Ambient condition synthesis of directly conjugated nanoparticle to protein has been attempted by the reduction of disulfide bond ${ }^{11}$ with sodium borohydride $\left(\mathrm{NaBH}_{4}\right)$. Because $\mathrm{NaBH}_{4}$ is a strong reducing agent, ${ }^{12}$ usage of this may rupture any disulfide bond resulting in nonspecific binding of nanoparticle to the protein.

In the present study, we have used a mild reducing agent TCEP (tris (2-carboxyethyl)phosphine hydrochloride) to reduce specifically ${ }^{13}$ the solvent exposed disulfide bonds of a transporter protein human serum albumin (HSA) at $\mathrm{pH} 4.5$. Semiconductor nanocrystal $\mathrm{CdS}$ has been grown amid the reduced disulfide linkage of the protein. The whole reaction has been carried out under an inert argon environment. Recently, such covalent attachment of the nanocrystal to an enzyme, $\alpha$-chymotrypsin, has been reported from our group. ${ }^{14}$ Moderate retardation of the enzymatic activity and insignificant perturbation of structural integrity of the enzyme upon attachment of the CdS nanocrystals reveal the merit of the covalent synthesis to probe the functionality of a protein in a physiological environment. Here, the covalently bound $\mathrm{CdS}$ quantum dot has been employed for the first time as an optical Förster resonance energy transfer (FRET) probe for the detection of protein-folding intermediates. In recent years, significant efforts have been made to explore structural and dynamical evolution in the protein-folding pathway using FRET between organic dye molecules (see recent reviews $\left.{ }^{15,16}\right)$. One of the potential problems of using organic fluorophores in the FRET experiments is that they finally fall victim to the unavoidable photobleaching. ${ }^{16}$ Moreover, conventional organic dyes having relatively faster excited-state lifetime are inefficient to monitor slow protein-folding reactions. As compared to the conventional organic fluorophores/dyes, quantum dots have several advantageous properties, for example, tunable emission, exceptional photostability, higher photoluminescence lifetime, and higher quantum yield. ${ }^{17,18}$ FRET technique $^{19,20}$ has been employed to characterize the thermally 

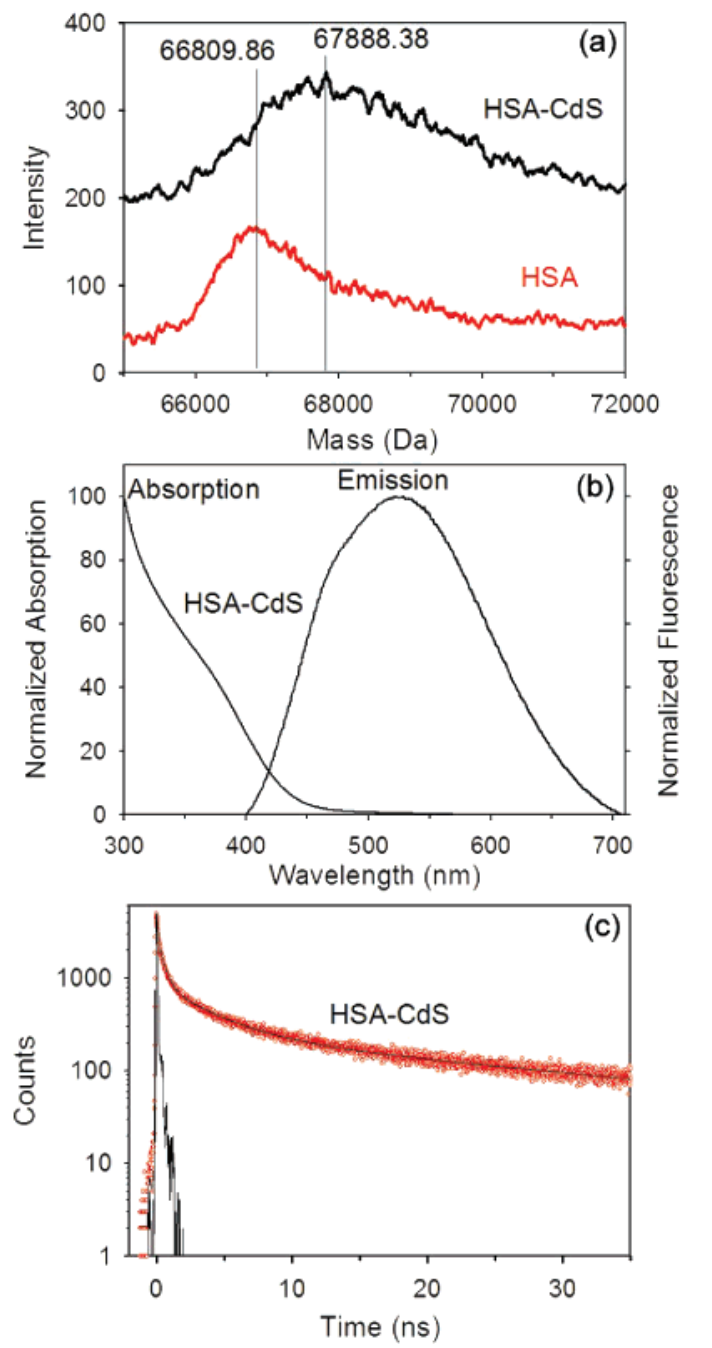

Figure 1. (a) MALDI-mass spectrum of HSA and CdS-labeled HSA. (b) UV-Vis absorption and photoluminescence spectra of CdS in HSACdS. (c) Picosecond-resolved photoluminescence transient of CdS in HSA (excitation wavelength $375 \mathrm{~nm}$ and detection wavelength $510 \mathrm{~nm}$ ).

unfolded states of HSA using this covalently attached new biological nanoprobe.

\section{Materials and Methods}

Human serum albumin (HSA), anhydrous $\mathrm{Na}_{2} \mathrm{~S} \cdot 9 \mathrm{H}_{2} \mathrm{O}, \mathrm{Cd}-$ $\left(\mathrm{NO}_{3}\right)_{2} \cdot 4 \mathrm{H}_{2} \mathrm{O}$, and TCEP (tris (2-carboxyethyl)phosphine hydrochloride)) were purchased from Sigma. The chemicals and the proteins were of highest commercially available purity and were used as received. All sample solutions have been prepared with deionized water ( $\mathrm{pH} 4.5)$ and completely degassed by dry argon. The aqueous solution of HSA was exhaustively dialyzed against water of $\mathrm{pH} 4.5$ at $4{ }^{\circ} \mathrm{C}$ to remove all additional salts from the protein. The disulfide bonds of HSA were reduced by injecting TCEP solution into the dialyzed protein solution (200 $\mu \mathrm{M})$ and vigorously stirring for $30 \mathrm{~min}$. It is well known ${ }^{12}$ that the reduction of solvent-exposed disulfides by TCEP proceeds readily at low $\mathrm{pH}$. The $\mathrm{pH}$ of HSA solution was maintained at 4.5 because TCEP reduces disulfides rapidly and cleanly in water at $\mathrm{pH}=4.5$ at room temperature. At these values of $\mathrm{pH}$, thiolate-disulfide interchange is effectively prevented because only low levels of thiolate are present. It is therefore possible to generate thiol from disulfide with kinetic selectivity under conditions in which thiolate-disulfide interchange does not occur. At the TCEP concentration we have used $(3 \mathrm{mM})$, the (a)
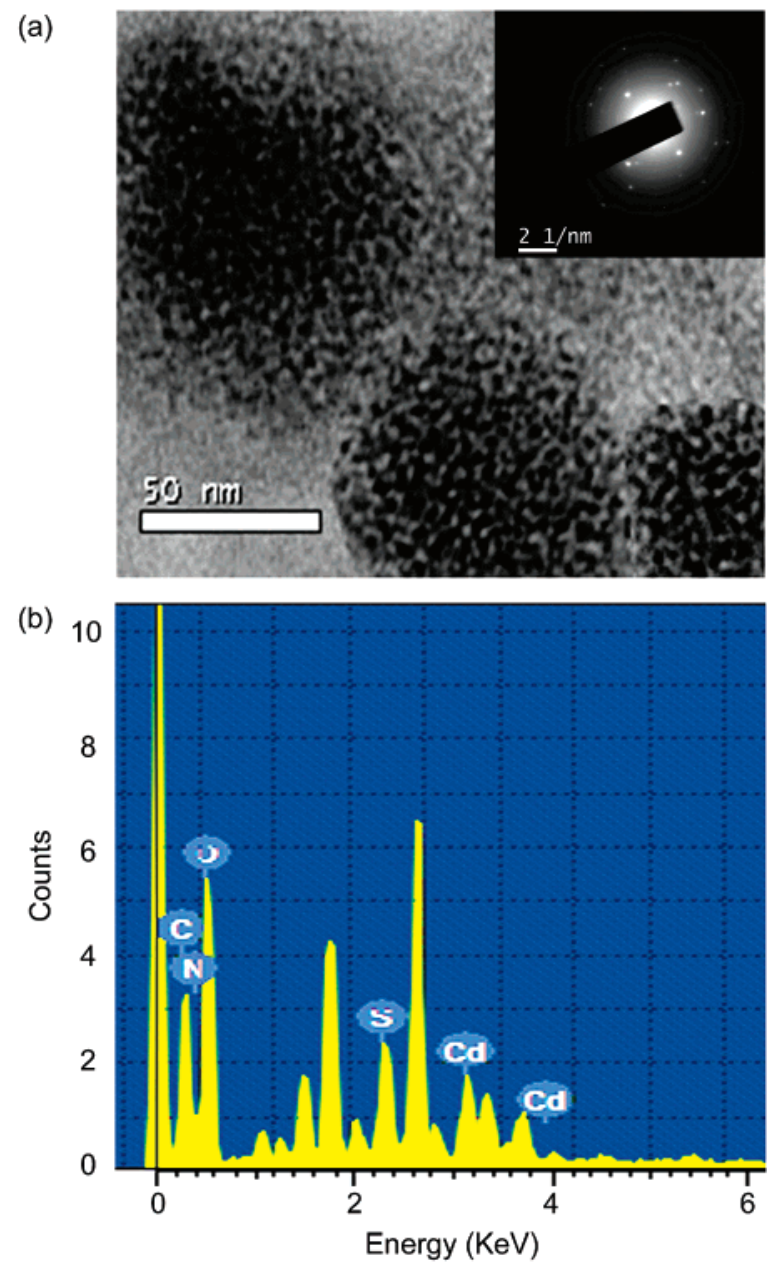

Figure 2. (a) High-resolution transmission electron micrograph (HRTEM) of CdS covalently bound to HSA. Inset shows selected area electron diffraction pattern (SAED) of CdS attached to the globular protein HSA. (b) Energy dispersive X-ray (EDAX) spectra of HSACdS.

reduction of disulfides was complete within $5 \mathrm{~min}$. A degassed aqueous $\mathrm{Cd}^{2+}$ solution $(1 \mathrm{M})$ was then added to the above solution (containing HSA and TCEP) and vigorously stirred for $30 \mathrm{~min}$. This was followed by dropwise addition of aqueous $\mathrm{S}^{2-}$ solution $(0.5 \mathrm{M})$ with vigorous stirring. The final concentration of $\mathrm{Cd}^{2+}$ and $\mathrm{S}^{2-}$ in the aqueous solution was maintained to be 20 and $10 \mathrm{mM}$, respectively. The reaction was allowed to proceed for $3 \mathrm{~h}$, and the final solution was centrifuged. The resultant supernatant was filtered and then dialyzed against water of $\mathrm{pH}=4.5$ at $4{ }^{\circ} \mathrm{C}$ to remove any excess of salts and TCEP left in the solution. The final dialyzed solution was collected and stored at $4{ }^{\circ} \mathrm{C}$ prior to analysis. The donor-acceptor (DA) distance $(r)$ was measured by the following equation: ${ }^{19}$

$$
r=\left[\frac{R_{0}^{6}(1-E)}{E}\right]^{1 / 6}
$$

Here, $R_{0}$ and $E$ are the Förster distance and the donor efficiency of energy transfer, respectively. The details of experimental techniques used can be obtained from the Supporting Information.

\section{Results and Discussion}

The covalent binding of CdS to HSA is confirmed by matrixassisted laser desorption ionization (MALDI)-mass spectrometry analysis (Figure 1a). A peak at $66809.86 \mathrm{Da}$ corresponding to 
SCHEME 1: Three-Dimensional Structure of $\mathbf{H S A}^{a}$

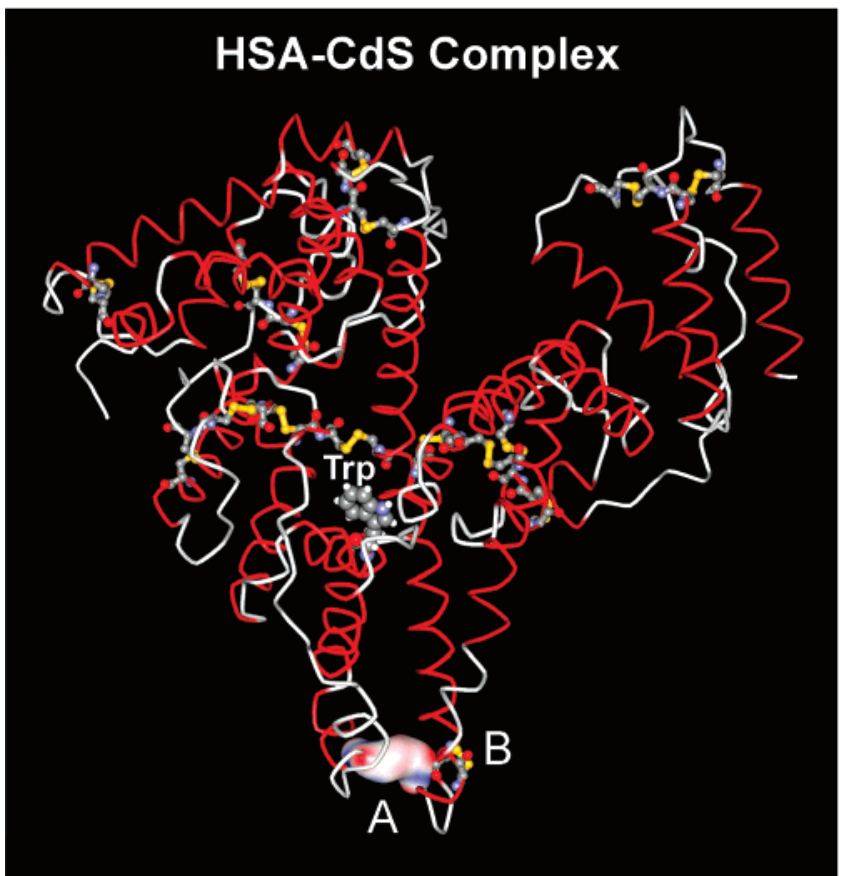

The location of tryptophan 214 (Trp) is indicated. The possible sites for CdS attachment, sites A and B, essentially indicate the positions of the Cys316-Cys361 and Cys360-Cys369 disulfide bonds, respectively. Nucleation of the nanocrystal is likely to occur in site A (see text). The solvent-accessible surface area (SASA) of site A is also shown. Ball-stick models are used to indicate other disulfide bonds of the protein. The coordinates of HSA structure were downloaded from the Protein Data Bank (PDB code 1UOR) and processed with the WebLab Viewer Lite program.

the molecular mass of unlabeled HSA shifts to $67888.38 \mathrm{Da}$ for the CdS-labeled HSA. The increase of the mass of HSA$\mathrm{CdS}$ nanocomposites of $1078.52 \mathrm{Da}$ is equivalent to $7.5 \mathrm{CdS}$ (formula weight 144) molecules (15 atoms) in the nanocrystal conjugated to HSA. Theoretical estimation ${ }^{21}$ of the diameter of the CdS nanocrystals of 15 atoms reveals a value of $\sim 1 \mathrm{~nm}$ on considering the crystal structure to be wurzite (see below). The absorption spectrum of HSA-CdS exhibits a band $\sim 370 \mathrm{~nm}$ (Figure 1b), which indicates the presence of a quantized state of CdS nanoparticles. The blue-shifted absorption band with respect to the bulk CdS $(\sim 515 \mathrm{~nm})$ is indicative of quantum confinement effect in the nanoparticles. The particle diameter and band gap are estimated to be $1.26 \mathrm{~nm}$ and $3.38 \mathrm{eV}$, respectively. ${ }^{22,23}$ The observed photoluminescence band (Figure 1b) centered at $528 \mathrm{~nm}$ is attributed to the recombination of charge carriers within surface states, which is also observed in the picosecond-resolved fluorescence transients (Figure 1c). The quantum yield of $\mathrm{HSA}-\mathrm{CdS}$ at $25{ }^{\circ} \mathrm{C}$ is 0.49 . When the temperature increases to $75^{\circ} \mathrm{C}$, the quantum yield changes to 0.37 . The time-resolved transient (laser excitation $=375 \mathrm{~nm}$, detection at $510 \mathrm{~nm}$ ) is multiexponential in nature with four decay time components with various pre-exponential values of 56 ps (73\%), 420 ps (19\%), $3.61 \mathrm{~ns}(6 \%)$, and $30.77 \mathrm{~ns}(2 \%)$. The faster lifetime is due to nonradiative carrier relaxation from intrinsic to underlying trap states. ${ }^{24-26}$ The relatively slower time constants $(>100 \mathrm{ps})$ may have some relevance to the phonon bottleneck effect. ${ }^{26}$ On the other hand, the slowest component associated with band edge emission ${ }^{24}$ exceeds the time window of our experimental setup.

A high-resolution transmission electron microscopy (HRTEM) image of HSA-CdS is presented in Figure 2a. A statistical analysis of $\sim 200$ particles (by hand) yields an average particle
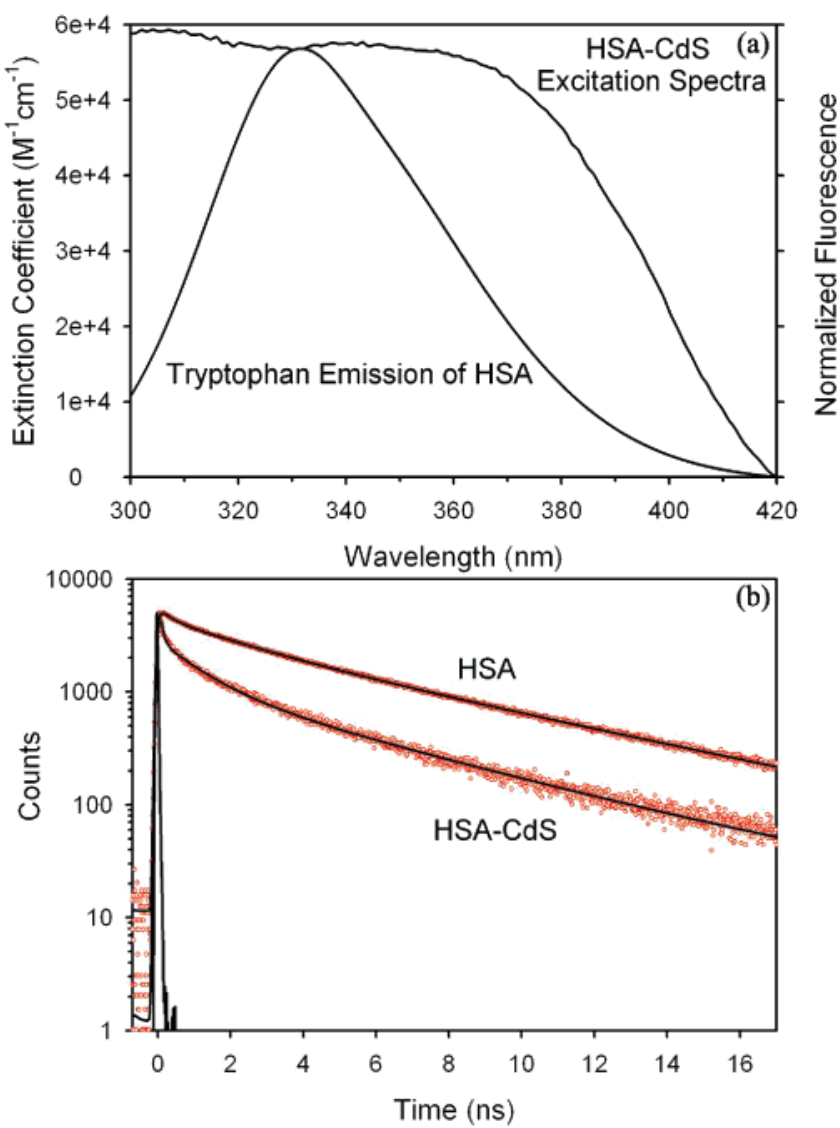

Figure 3. (a) Spectral overlap between the donor (tryptophan in HSA, Trp214) emission and acceptor (CdS tagged with HSA) excitation. (b) Picosecond-resolved fluorescence transients of Trp214 in HSA (excitation wavelength $299 \mathrm{~nm}$ and detection wavelength $360 \mathrm{~nm}$ ) in the absence and presence of CdS quantum dots in aqueous solution.

size of $1.5 \pm 0.3 \mathrm{~nm}$. The selected area electron diffraction (SAED) pattern (inset of Figure 2a) suggests single crystalline structure. The pattern shows diffraction spots at $d$ spacings of $2.44,2.02$, and $1.35 \AA$, which are assignable to diffractions from (102), (110), and (203) planes of a hexagonal crystal structure of $\mathrm{CdS},{ }^{27}$ respectively. Energy dispersive X-ray analysis (EDAX) of HSA-CdS (Figure 2b) confirms the presence of $\mathrm{Cd}$ and $\mathrm{S}$ with an atomic ratio of $2: 1$. The observation of relatively higher diameter of the CdS particles as revealed from absorption, TEM, and EDAX studies as compared to that of the estimated value from mass spectroscopy may suggest that excess $\mathrm{Cd}^{2+}$ ions are noncovalently attached to the surface of the $\mathrm{CdS}$ nanocrystal.

The stereoview of HSA is shown in Scheme 1. HSA, the most abundant protein of blood plasma, is formed from a single polypeptide chain of 585 residues, and the whole structure is divided into three similar globular domains (I-III), each of which contains two (A, B) subdomains. ${ }^{28}$ The single tryptophan Trp214 of HSA resides in domain IIA. One of the 17 disulfide bonds of HSA is supposed to react with the reducing agent TCEP to host $\mathrm{CdS}$, as the molar ratio of protein:CdS is estimated to be 1:1 from UV-Vis absorption studies. To locate the disulfide bond/bonds responsible to host $\mathrm{CdS}$ nanocrystal, Trp214 to CdS nanocrystal resonance energy transfer is examined. The huge spectral overlap of Trp214 emission with the absorption of CdS nanocrystal is expected to reveal interprobe distance, when they are in a close proximity. Figure $3 \mathrm{a}$ shows the spectral overlap between the tryptophan emission (donor D, emission maximum at $332 \mathrm{~nm}$ ) and $\mathrm{CdS}$ excitation (acceptor A, excitation maximum at $370 \mathrm{~nm}$ ) spectra in HSA at room temperature. The quenching of donor emission is 

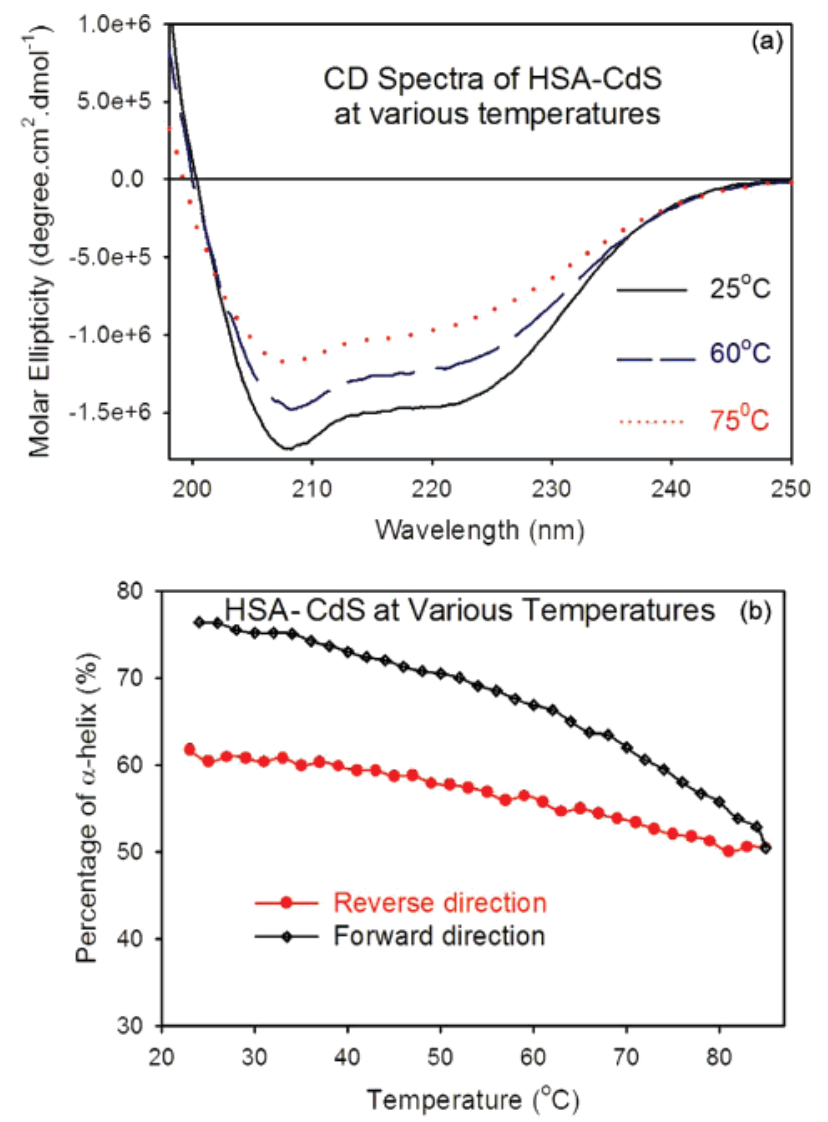

Figure 4. (a) Circular dichroism (CD) spectra of HSA-CdS in water ( $\mathrm{pH} \mathrm{4.5)}$ at three temperatures. (b) Percentage of $\alpha$-helix content in CdS tagged HSA as a function of temperature.

evident from the faster lifetime (excitation source $=299 \mathrm{~nm}$ ) associated with the donor-acceptor pair (HSA labeled with CdS nanocrystal) as compared to that in the donor alone (unlabeled HSA) (Figure $3 \mathrm{~b}$ and Table 1). The D-A distances ( $r$ ) obtained from FRET experiments with varying temperature are summarized in Table 1. The average $\mathrm{D}-\mathrm{A}$ distance at room temperature is found to be $26.1 \AA$. The measured distance indicates that the possible locations of CdS are close to either Cys316-Cys361 (distance from Trp214 = 27.4 ̊) or Cys360Cys369 (distance from Trp214= $27.5 \AA$ ), which are in subdomain IIB and significantly exposed to the solvent environment, ${ }^{28}$ as indicated by sites A and B of Scheme 1 . We have further estimated the solvent-accessible surface area (SASA) of the two sites of HSA using the WebLab viewer Lite program, which was found to have values of 184.6 and $179.1 \AA^{2}$, respectively. Higher value of SASA of the site A as compared to that of the site $\mathrm{B}$ makes the former site more favorable for $\mathrm{CdS}$ attachment.
After characterizing the attachment of CdS to HSA using FRET technique, which essentially probes the inter subdomain distance between IIA (Trp214 position) and IIB (CdS position), we have used the distance as a marker of various temperatureinduced folded states of the protein. Temperature-induced unfolded states of the protein avoid complications due to chemical denaturation. As evidenced from Table 1 with the increase in temperature from 25 to 60 and finally to $75^{\circ} \mathrm{C}$, the average $\mathrm{D}-\mathrm{A}$ distance changes, which is the signature of swelling of the protein. Refolding of the protein upon subsequent cooling to $25^{\circ} \mathrm{C}$ that resulted in the recovery of $\mathrm{D}-\mathrm{A}$ distances does not follow a similar trend. These observations are consistent with one of our dynamic light scattering (DLS) studies on the protein HSA. ${ }^{29}$ The unfolding of a protein is marked by the alteration of secondary structure of the protein. The conformational transition of $\mathrm{HSA}^{30}$ and $\mathrm{HSA}-\mathrm{CdS}$ with temperature follows a similar pathway. Circular dichroism (CD) spectroscopic measurements show the change in the mean residue ellipticity of the HSA-CdS at temperatures of 25, 60, and $75^{\circ} \mathrm{C}$, respectively (Figure $4 \mathrm{a}$ ). The percentage of $\alpha$-helix in the CdS tagged HSA, indicated by the negative peaks at 208 and $220 \mathrm{~nm}$, decreases from $76.4 \%$ in the native state at $25^{\circ} \mathrm{C}$ to $66.9 \%$ at $60{ }^{\circ} \mathrm{C}$ and $58.8 \%$ at $75^{\circ} \mathrm{C}$ (Figure 4b). The helix content changes from $69.7 \%$ at $25^{\circ} \mathrm{C}$ to $58.4 \%$ at $60{ }^{\circ} \mathrm{C}$ to $45.9 \%$ at $75{ }^{\circ} \mathrm{C}$ for unlabeled HSA at $\mathrm{pH} 4.5$ (Supporting Information Figure S1). This shows that the protein loses a considerable fraction of the secondary structure in thermal denaturation. The decrease in the percentage of $\alpha$-helix of HSA$\mathrm{CdS}$ is shown as a function of temperature in Figure $4 \mathrm{~b}$, which is consistent with an earlier report. ${ }^{31}$ The plots reveal that the increase in percentage helicity upon thermal cooling from $75{ }^{\circ} \mathrm{C}$ to $25{ }^{\circ} \mathrm{C}$ is not fully reversible. The existence of intermediate states in the thermal unfolding pathway also becomes evident from the changes in secondary structure.

One of the early studies ${ }^{31}$ on the covalently labeled acrylodan to HSA reveals that thermal unfolding of the protein HSA follows at least three distinct steps: native $\rightarrow$ extended $\rightarrow$ intermediate $\rightarrow$ unfolded state. The study shows that, by increasing the temperature to less than $50{ }^{\circ} \mathrm{C}$, reversible separation of domain I and II results. At temperature above $60{ }^{\circ} \mathrm{C}$, irreversible unfolding/melting of domain II happens, whereas domain I remains intact. In the present study, emission of Trp214 is used to examine domain IIA. The observed results are consistent with the above temperature-dependent protein-folding studies. ${ }^{31}$ Our study of thermal unfolding of HSA using CdS nanocrystal is also in agreement with the circular dichroism study of HSA and the study by Wetzel et al. ${ }^{30}$ that reveals $\alpha$-helical content of HSA upon thermal unfolding to $75^{\circ} \mathrm{C}$ following cooling to $25^{\circ} \mathrm{C}$ is unable to refold into the native form.

TABLE 1: Helix Content, Picosecond-Resolved Transients, and Energy-Transfer Parameters of HSA before and after Labeling with CdS Nanocrystal ${ }^{a}$

\begin{tabular}{|c|c|c|c|c|c|c|c|c|}
\hline systems & temperature & $\begin{array}{c}\alpha \text {-helix } \\
\text { content }(\%)\end{array}$ & $\tau_{1}$ in ns (\%) & $\tau_{2}$ in ns (\%) & $\tau_{3}$ in ns (\%) & $Q_{\mathrm{D}}$ & $R_{0}(\AA)$ & $r(\AA)$ \\
\hline \multirow[t]{5}{*}{ HSA } & $25^{\circ} \mathrm{C}$ & 69.7 & $0.23(18.1)$ & $1.65(23.6)$ & $5.99(58.3)$ & & & \\
\hline & $60^{\circ} \mathrm{C}$ & 58.4 & $0.15(29.4)$ & $1.46(31.3)$ & $4.56(39.3)$ & & & \\
\hline & $75^{\circ} \mathrm{C}$ & 45.9 & $0.11(40.8)$ & $1.15(32.6)$ & $3.82(26.6)$ & & & \\
\hline & $60{ }^{\circ} \mathrm{C}$ (reverse) & 49.4 & $0.09(45.5)$ & $1.08(30.3)$ & $3.69(24.2)$ & & & \\
\hline & $25^{\circ} \mathrm{C}$ (reverse) & 60.5 & $0.13(36.7)$ & $1.23(30.0)$ & $4.63(33.3)$ & & & \\
\hline \multirow[t]{5}{*}{ HSA-CdS } & $25^{\circ} \mathrm{C}$ & 76.4 & $0.08(73.8)$ & $1.45(16.6)$ & $5.99(9.6)$ & 0.097 & 32.1 & 26.1 \\
\hline & $60^{\circ} \mathrm{C}$ & 66.9 & $0.08(72.4)$ & $1.12(20.6)$ & $4.56(7.0)$ & 0.092 & 32.9 & 27.7 \\
\hline & $75^{\circ} \mathrm{C}$ & 58.8 & $0.07(69.8)$ & $0.92(24.0)$ & $3.82(6.2)$ & 0.087 & 31.6 & 28.6 \\
\hline & $60{ }^{\circ} \mathrm{C}$ (reverse) & 55.2 & $0.06(72.9)$ & $0.89(20.0)$ & $3.69(7.1)$ & 0.091 & 33.0 & 30.4 \\
\hline & $25^{\circ} \mathrm{C}$ (reverse) & 60.4 & $0.05(75.9)$ & $1.02(15.3)$ & $4.63(8.8)$ & 0.091 & 33.0 & 28.8 \\
\hline
\end{tabular}

${ }^{a}$ Here, $Q_{\mathrm{D}}, R_{0}$, and $r$ denote quantum yield of the donor in the absence of the acceptor, Förster distance, and D-A distance, respectively. 


\section{Conclusions}

In conclusion, we have synthesized luminescent semiconductor nanocrystals covalently attached to a transporter protein HSA, and the fluorescent dots are found to be an efficient probe, to monitor the intermediates of protein folding. It has to be noted that the size of the QD in our studies is $\sim 1 \mathrm{~nm}$ ( 15 atoms), which is similar to that of the conventional organic dyes. The interference of the larger sized QD motion with protein folding is thus avoided. The FRET technique used in the present study employs one of the intrinsic fluorescent amino acids Trp214 of HSA as a donor, which has significant spectral overlap with that of the acceptor, CdS nanocrystal, at the Cys316-Cys361 site. However, the methodology can be used for other extrinsic fluorophores including luminescent QDs of variable sizes as energy donors. The study is expected to open new insight into material science research to the direct application of quantum dots to the field of medicinal chemistry and biology.

Acknowledgment. R.S. thanks UGC and S.S.N. thanks CSIR for fellowships. We thank DST, India (SR/FTP/PS-05/ 2004), and British Council (UKIERI) for a financial grant.

Supporting Information Available: Details of steady-state measurements, time-resolved measurements, and donor-acceptor distance measurements from FRET, estimation of nanocrystals size, references, and Figure $\mathrm{S} 1$. This material is available free of charge via the Internet at http://pubs.acs.org.

\section{References and Notes}

(1) LaVan, D. A.; Lynn, D. M.; Langer, R. Nat. Rev. Drug Discovery 2002, 1, 77-84.

(2) Niemeyer, C. M. Angew. Chem., Int. Ed. 2001, 40, 4128-4158.

(3) Stone, J. W.; Sisco, P. N.; Goldsmith, E. C.; Baxter, S. C.; Murphy, C. J. Nano Lett. 2007, 7, 116-119.

(4) Bradley, J. S.; Schmid, G. Clusters and Colloids: From Theory to Applications; VCH: Weinheim, 1994.

(5) Fu, A.; Gu, W.; Boussert, B.; Koski, K.; Gerion, D.; Manna, L.; Le Gros, M.; Larabell, C. A.; Alivisatos, A. P. Nano Lett. 2007, 7, 179182.

(6) Mamedova, N. N.; Kotov, N. A.; Rogach, A. L.; Studer, J. Nano Lett. 2001, 1, 281-286.
(7) Mirkin, C. A.; Letsinger, R. L.; Mucic, R. C.; Storhoff, J. J. Nature 1996, 382, 607-609.

(8) Gao, X.; Chan, W. C. W.; Nie, S. J. Biomed. Opt. 2002, 7, 532537.

(9) Meziani, M. J.; Pathak, P.; Harruff, B. A.; Hurezeanu, R.; Sun, Y.-P. Langmuir 2005, 21, 2008-2011.

(10) Meziani, M. J.; Sun, Y.-P. J. Am. Chem. Soc. 2003, 125, 80158018.

(11) Burt, J. L.; Gutierrez-Wing, C.; Miki-Yoshida, M.; Jose-Yacaman, M. Langmuir 2004, 20, 11778-11783.

(12) Haugland, R. P. Handbook of Fluorescent Probes and Research Chemicals, 7th ed.; Molecular Probes: Eugene, OR, 1996.

(13) Burns, A. J.; Butler, J. C.; Moran, J.; Whitesides, G. M. J. Org Chem. 1991, 56, 2648-2650.

(14) Narayanan, S. S.; Sarkar, R.; Pal, S. K. J. Phys. Chem. C 2007, 111, 11539-11543.

(15) Jäger, M.; Nir, E.; Weiss, S. Protein Sci. 2006, 15, 640-646.

(16) Nienhaus, G. U. Macromol. Biosci. 2006, 6, 907-922.

(17) Michalet, X.; Pinaud, F. F.; Bentolila, L. A.; Tsay, J. M.; Doose, S.; Li, J. J.; Sundaresan, G.; Wu, A. M.; Gambhir, S. S.; Weiss, S. Science 2005, 307, 538-544.

(18) Lakowicz, J. R.; Gryczynski, I.; Gryczynski, Z.; Nowaczyk, K.; Murphy, C. J. Anal. Biochem. 2000, 280, 128-136.

(19) Lakowicz, J. R. Principles of Fluorescence Spectroscopy; Kluwer Academic/Plenum: New York, 1999.

(20) Sarkar, R.; Shaw, A. K.; Narayanan, S. S.; Dias, F.; Monkman, A.; Pal, S. K. Biophys. Chem. 2006, 123, 40-48.

(21) Viswanatha, R.; Sapra, S.; Satpati, B.; Satyam, P. V.; Dev, B. N. Sarma, D. D. J. Mater. Chem. 2004, 14, 661-668.

(22) Brus, L. E. J. Chem. Phys. 1984, 80, 4403-4409.

(23) Demchenko, D. O.; Wang, L.-W. Phys. Rev. B 2006, 73, 155326.

(24) Burda, C.; Link, S.; Mohamed, M.; El-Sayed, M. J. Phys. Chem. B 2001, 105, 12286-12292.

(25) Burda, C.; Chen, X.; Narayanan, R.; El-Sayed, M. A. Chem. Rev. 2005, 105, 1025-1102.

(26) Sarkar, R.; Shaw, A. K.; Narayanan, S. S.; Rothe, C.; Hintschich, S.; Monkman, A.; Pal, S. K. Opt. Mater. 2007, 29, 1310-1320.

(27) Cheng, Y.; Wang, Y.; Chen, D. J. Phys. Chem. B 2006, 110, $9448-$ 9451.

(28) He, X. M.; Carter, D. C. Nature 1992, 358, 209-215.

(29) Mitra, R. K.; Sinha, S. S.; Pal, S. K. Langmuir 2007, 23, 1022410229

(30) Wetzel, R.; Becker, M.; Behlke, J.; Wite, H.; Bohn, S.; Ebert, B.; Hamaann, H.; Krumbiegel, J.; Lasiman, G. Eur. J. Biochem. 1980, 104, 469-478.

(31) Flora, K.; Brennan, J. D.; Baker, G. A.; Doody, M. A.; Bright, F. V. Biophys. J. 1998, 75, 1084-1096. 


\section{$\underline{\text { Supplementary Material }}$}

\section{Direct Conjugation of semiconductor nanocrystals to a globular protein to study protein-folding intermediates}

Rupa Sarkar ${ }^{1}$, S. Shankara Narayanan ${ }^{1}$, Lars-Olof Palsson ${ }^{2}$, Fernando Dias ${ }^{2}$, Andy Monkman $^{2}$ and Samir Kumar Pal ${ }^{1, *}$

${ }^{1}$ Unit for Nano Science \& Technology, Department of Chemical, Biological \& Macromolecular Sciences, S. N. Bose National Centre for Basic Sciences, Block JD, Sector III, Salt Lake, Kolkata 700 098, India. ${ }^{2}$ Department of Physics, University of Durham, UK.

*Corresponding Author. Email: skpal@ bose.res.in; Fax: 913323353477. 
Steady State Measurements (S1): Temperature dependent steady-state absorption and emission are measured with Shimadzu Model UV-2450 spectrophotometer and Jobin Yvon Fluoromax-3 fluorimeter respectively with a temperature controller attachment from Julabo (Model: F32). A JEOL JEM-2100 high-resolution transmission electron microscope (HRTEM) equipped with an energy dispersive X-ray (EDAX) spectrometer is used to characterize the microscopic structures of samples and to analyze their elemental composition. The size of the nanoparticles is determined from the TEM images obtained at $200 \mathrm{kV}$. Sample for TEM is prepared by placing a drop of the HSA-CdS solution on a carbon-coated copper grid and then allowing it to evaporate overnight at room temperature. Matrix-assisted laser desorption ionization (MALDI) mass spectra are obtained using an Applied Biosystem Voyager-DE PRO mass spectrometer. The circular dichroism (CD) measurements are done in a JASCO 815 spectropolarimeter with a temperature controller attachment (Peltier). For CD experiments, the protein concentration and cell path-length are maintained at $1.0 \mu \mathrm{M}$ and $1 \mathrm{~cm}$ respectively. The secondary structural data of the CD spectra are analyzed using CDNN software. CD spectrum of HSA without CdS is attached (figure S1) to show any alteration of the protein secondary structure due to conjugation of the CdS nanocrystal.

Time resolved measurements (S2): All fluorescence transients are measured and have been fitted by using commercially available picosecond diode laser-pumped (LifeSpec-ps) time-resolved fluorescence spectrophotometer from Edinburgh Instruments, U.K. with a temperature controller attachment for temperature dependent studies. The picosecond excitation pulse from the picoquant diode laser was used at $375 \mathrm{~nm}$ (instrument response function, IRF of $80 \mathrm{ps}$ ) in order to excite CdS QD. For the excitation of tryptophan in 
HSA (Trp214) we have used excitation wavelength $299 \mathrm{~nm}$. The excitation source (299nm) consisted of a mode-locked cavity dumped (APETM Pulse switch) Ti:Saphire laser (MIRA ${ }^{\mathrm{TM}}$, Coherent). Near infrared optical pulses with a temporal width of $\sim 200 \mathrm{fs}$ (FWHM) was used to generate the 3rd harmonic generation UV pulses at wavelength of $\sim 299 \mathrm{~nm}$ with an Inrad ${ }^{\mathrm{TM}}$ (Model 5-050) harmonic generator. The fluorescence was detected using a photomultiplier tube (id Qunatic ${ }^{\mathrm{TM}}$ Model id 100-50) linked to a timeto-amplitude converter (Ortec ${ }^{\mathrm{TM}}$ 567) and multichannel analyzer (E.G. \& G ${ }^{\mathrm{TM}}$. Trump Card and Maestro for Windows v. 5.10). The IRF of the apparatus was measured from the Rayleigh scattered light, giving an IRF with a duration of 60 ps FWHM. All fluorescence decays were recorded to a minimum of 5,000 counts in the peak channel of the pulse height analyzer. The observed fluorescence transients are fitted by using a nonlinear least square fitting procedure to a function $\left(X(t)=\int_{0}^{t} E\left(t^{\prime}\right) R\left(t-t^{\prime}\right) d t^{\prime}\right)$ comprising of convolution of the IRF $(\mathrm{E}(\mathrm{t}))$ with a sum of exponentials $\left(\mathrm{R}(\mathrm{t})=\mathrm{A}+\sum_{\mathrm{i}=1}^{\mathrm{N}} \mathrm{B}_{\mathrm{i}} \mathrm{e}^{-\mathrm{t} / \tau_{\mathrm{i}}}\right)$ with pre-exponential factors $\left(\mathrm{B}_{\mathrm{i}}\right)$, characteristic lifetimes $\left(\tau_{\mathrm{i}}\right)$ and a background (A). Relative concentration in a multi-exponential decay is finally expressed as; $c_{n}=\frac{B_{n}}{\sum_{i=1}^{N} B_{i}} \times 100$. The quality of the curve fitting is evaluated by reduced chi-square and residual data.

Donor-Acceptor distance Measurement from FRET (S3): In order to estimate the Förster resonance energy transfer efficiency (FRET) of the donor (amino acid tryptophan of HSA) to the acceptor (CdS nanocrystal in HSA) and hence to determine distances of 
donor-acceptor pairs, we have followed the methodology described in chapter 13 of reference ${ }^{1}$ and reference ${ }^{2}$. The Förster distance $\left(\mathrm{R}_{0}\right)$ is given by,

$$
\mathrm{R}_{0}=0.211\left[\kappa^{2} \mathrm{n}^{-4} \mathrm{Q}_{\mathrm{D}} \mathrm{J}(\lambda)\right]^{1 / 6} \quad \text { (in } \AA \text { ) }
$$

where $\kappa^{2}$ is a factor describing the relative orientation in space of the transition dipoles of the donor and acceptor. For donor and acceptors that randomize by rotational diffusion prior to energy transfer, the magnitude of $\kappa^{2}$ is assumed to be $2 / 3$. In the present study the same assumption has been made. The refractive index $(n)$ of the medium is assumed to be 1.4. $Q_{\mathrm{D}}$ is the the quantum yield of the donor in the absence of acceptor. $\mathrm{J}(\lambda)$, the overlap integral, which expresses the degree of spectral overlap between the donor emission and the acceptor absorption, is given by,

$$
\mathrm{J}(\lambda)=\frac{\int_{0}^{\infty} F_{D}(\lambda) \varepsilon(\lambda) \lambda^{4} d \lambda}{\int_{0}^{\infty} \mathrm{F}_{\mathrm{D}}(\lambda) d \lambda}
$$

where $F_{D}(\lambda)$ is the fluorescence intensity of the donor in the wavelength range of $\lambda$ to $\lambda+\mathrm{d} \lambda$ and is dimensionless. $\varepsilon(\lambda)$ is the extinction coefficient (in $\mathrm{M}^{-1} \mathrm{~cm}^{-1}$ ) of the acceptor at $\lambda$. If $\lambda$ is in $\mathrm{nm}$, then $\mathrm{J}(\lambda)$ is in units of $\mathrm{M}^{-1} \mathrm{~cm}^{-1} \mathrm{~nm}^{4}$. Once the value of $\mathrm{R}_{\mathrm{o}}$ is known, the donor-acceptor distance (r) can easily be calculated using the formula,

$$
r^{6}=\left[R_{0}{ }^{6}(1-E)\right] / E .
$$

Here $\mathrm{E}$ is the efficiency of energy transfer. The transfer efficiency (E) is measured using the relative lifetime of the donor in the absence $\left(\tau_{\mathrm{D}}\right)$ and presence $\left(\tau_{\mathrm{DA}}\right)$ of the acceptor.

$$
\mathrm{E}=1-\left(\tau_{\mathrm{DA}} / \tau_{\mathrm{D}}\right)
$$

In order to obtain lifetime of the donor tryptophan (Trp214) in absence of acceptor, we have measured tryptophan excited state lifetime of HSA (without CdS labeling). In the 
HSA-CdS complexes the time constants remain nearly similar to those of the protein without nanocrystals and have been made fixed during numerical fitting to avoid complicacy in the FRET analysis. The goodness of the fitting $\left(\chi^{2}\right)$ has also been checked for both the fixed and free fitting cases and it lies in the range of 0.93-1.08. The average time constants $\left(\sum_{\mathrm{i}} \mathrm{c}_{\mathrm{i}} \tau_{\mathrm{i}}\right)$ of $\operatorname{Trp} 214$ in the absence and presence of acceptor $\operatorname{CdS}$ nanocrystal are regarded as $\tau_{\mathrm{D}}$ and $\tau_{\mathrm{DA}}$.

\section{Estimation of nanocrystal size (S4):}

The effective diameter, $\mathrm{d}$, of the nanocrystal is calculated assuming that the particles are spherical in shape using the formula ${ }^{3}$

$$
\mathrm{d}=\left(\frac{3 N a^{2} c}{2 \pi}\right)^{1 / 3}
$$

where a and $\mathrm{c}$ are the bulk lattice parameters and $\mathrm{N}$ is the number of atoms in the nanocrystal. Selected area electron diffraction (SAED) measurement of HSA-CdS nanocrystal reveals the structure is hexagonal (wurtzite) and as CdS binds covalently to the protein, number of $\mathrm{CdS}$ atoms per nanocrystal is 15 and the diameter is calculated to be $9.32 \AA$ considering $a=4.12 \AA, c=6.65 \AA$.

\section{References:}

1. J. R. Lakowicz, Principles of fluorescence spectroscopy; Kluwer Academic/Plenum: New York, 1999.

2 R. Sarkar, A. K. Shaw, S. S. Narayanan, F. Dias, A. Monkman, S. K. Pal, Biophysical chemistry 2006, 123, 40.

3 R. Viswanatha, S. Sapra, B. Satpati, P. V. Satyam, B. N. Dev, D. D. Sarma, J. Mater. Chem. 2004, 14, 661. 

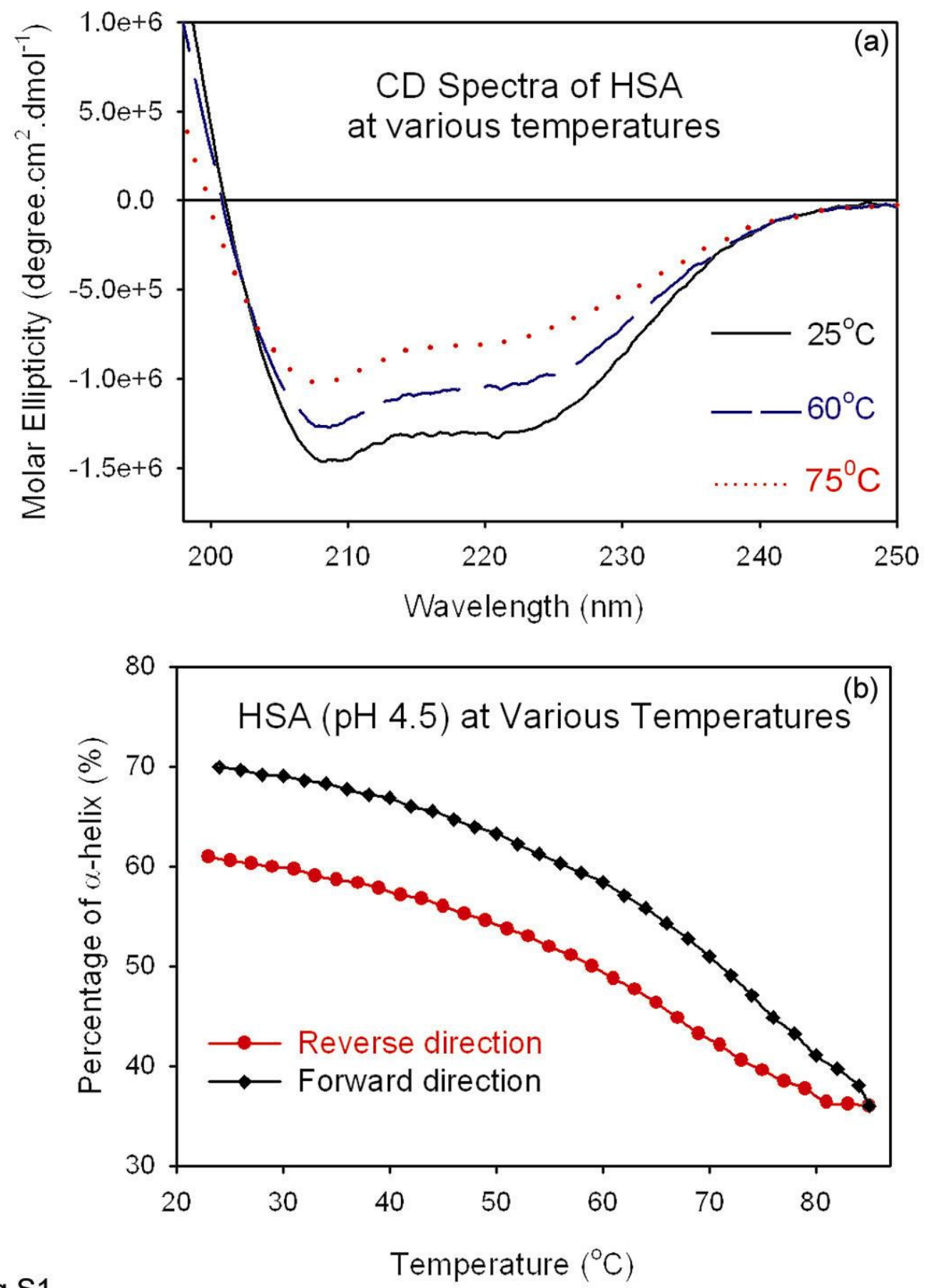

Fig.S1 